
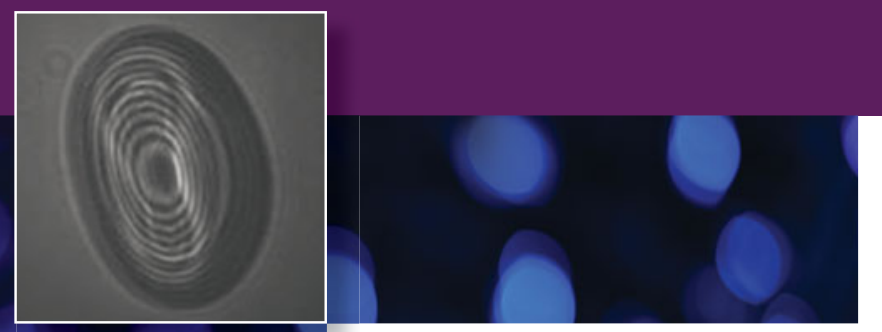

\title{
Advances and opportunities of ultrafast laser synthesis and processing
}

\author{
Steven M. Yalisove, Koji Sugioka, and Costas P. Grigoropoulos, \\ Guest Editors
}

\begin{abstract}
Ultrafast laser synthesis and processing of materials is a burgeoning field that is still in its infancy. This article and the theme articles in this issue review recent developments in the fundamental physics of ultrafast laser-solid interactions as well as the state of our understanding of ultrafast laser-driven surface morphology, modification of transparent media, 3D photo-polymerization and additive fabrication, spallation of graphene, and biological interactions. Also reviewed is the current state of emerging commercial high average power lasers, central to the widespread adoption of ultrafast laser synthesis and processing of materials. It is remarkable that ultrafast lasers with 20 to 600 femtosecond pulse duration can have such a dramatic impact on materials. As we learn more about the fundamental mechanisms that drive the ultrafast laser-material response, even more applications are anticipated to emerge. This revolutionary approach to materials synthesis and processing has already spawned several commercial technologies and promises to create many more in the near future.
\end{abstract}

\section{Introduction}

Our understanding of ultrafast laser interactions with materials has come a long way since MRS Bulletin published a theme issue on the topic 10 years ago. ${ }^{1}$ The most significant advances have resulted from our continued understanding of the atomistic mechanisms and dynamics that control the material response. Both theoretical and experimental work have contributed to these advances that are reviewed in the articles in this issue.

There is still much to learn about fundamental laser-solid interactions. We have only just scratched the surface, and tremendous opportunities exist to exploit the extreme conditions that are attainable with ultrafast laser interactions. New computational tools and powerful parallel processing ability will enable us to tackle time-dependent excited-state phenomena that are far from the ground-state Born-Oppenheimer surface.

Real opportunities in laser-solid interactions exist for the following reasons: (1) The ultrafast time scale can limit the material interaction with the laser field to only electronic excitation and leave the remaining ions with their roomtemperature velocity distribution; (2) the extreme conditions that a material can be brought to in temperature and pressure; (3) control of defect production at relatively low fluence; (4) capability of three-dimensional (3D) micro- and nanoprocessing; and (5) new opportunities in new materials classes, including biological and carbon-based materials.
A steady stream of applications has already resulted from our fundamental understanding of ultrafast laser-solid interaction. Some of these have achieved mainstream industrial adoption (e.g., femtosecond [fs] laser surgery ${ }^{2}$ ). If anything can be said about the 10 years since the last MRS Bulletin theme issue on ultrafast lasers and materials research, it is that there has been an explosion in the fundamental understanding (see the Shugaev et al. and Abere et al. articles in this issue), applications (see Jiang et al. in this issue), and technologies to develop tools for those applications (see Mottay et al. in this issue). Emerging nanoprocessing applications could take advantage of the high-resolution feature fabrication offered by ultrafast lasers coupled to scanning probes. ${ }^{3}$

Many challenges, however, remain before we can achieve the significant impact that ultrafast laser synthesis and processing promises. Many applications cannot be implemented on a commercial scale until higher average power lasers and, more importantly perhaps, both high peak power and high average power lasers with high stability and reliability are available. Current experimental and computational tools to study the earliest time scales continue to develop, but still need significant improvement. It has been clear throughout the history of ultrafast laser interactions of materials that what happens during the first few picoseconds (ps) does not stay in the first few ps. Rather, these short-lived events dictate a 
broad set of cascading mechanisms that can persist for microseconds and, in some cases, for seconds. ${ }^{4}$ These mechanisms can drive the final state of the material phase, morphology, microstructure, and composition. These changes can be permanent or reversible.

The articles in this issue cover broad aspects of ultrafast laser synthesis and processing, from the fundamental interactions at the shortest time scales to a wide range of applications. What may not be as evident in this theme is some of the other emerging opportunities that result from the extreme material states that can be driven by an ultrafast laser. This introductory article explores these opportunities.

\section{Electronic structure modification}

Early time scales for laser-solid interactions begin when intense electric (and magnetic, if relativistic intensities are reached) fields interact with the electrons in a solid. Precise control of the resulting electron energies and occupancy in states can be accomplished with the simple (in theory) parameters of laser intensity, pulse duration, and wavelength. Much has also been written about the opportunities to use temporal pulse shaping to induce chemistry ${ }^{5}$ - but these are usually at lower intensities than we are considering here; this issue is focused on how to ablate, melt, and introduce defects in materials.

When electrons acquire energy from an ultrafast laser pulse, they go into higher energy states in the unoccupied band structure of the material, or they leave the material entirely. These electrons acquire temperatures on the order of thousands of degrees Kelvin, while the ions remain near room temperature. This highly nonequilibrium state persists for as much as 10-20 ps, depending on the material. ${ }^{6,7}$

The interaction of the material with the laser will depend strongly on the type of bonding that is present in the material, and also depend on the intensity of the laser. Metals behave differently than semiconductors, which behave differently than insulators. Each of the different mechanisms of ablation can lead to interesting applications. The simplest to understand and the first to be developed is the interaction of ultrafast light with metals. Here, electrons are excited and then equilibrate, a few ps later, with the metal ions via electron phonon coupling. The earliest model for this was the two-temperature model, which continues to serve us well. ${ }^{8}$

When electrons equilibrate with the metal, they cause rapid heating upon electron-ion recombination. This leads to the phenomenon that is generally referred to as ultrafast melting in metals ${ }^{9}$ and semiconductors, ${ }^{10}$ as it is completely controlled by the electronic structure relaxation. Another way to think about ultrafast melting is that the ions lose their crystallinity before they acquire a Maxwellian velocity distribution. The so-called "non-thermal melting" may in fact happen in the few ps scale, as quantitatively shown in the x-ray pump and probe experiments. ${ }^{11}$ Subsequently, and in a slower transition in the tens of ps range, the ions can be heated up to temperatures above their melting or boiling point, depending on the excitation energy. The material melts and becomes liquid on time scales shorter than thermal expansion can begin. As a result, when expansion becomes dominant, a few ps after the laser has irradiated the material, the material expands rather violently and launches a shock wave into the material and a rarefaction wave away from the material. ${ }^{12}$ While the absorption depth of the laser is on the order of the skin depth of a metal, the electrons diffuse fast and deeper into the material to then transfer energy to the lattice system via collisions. The free volume in the liquid coalesces into voids about halfway through the molten region because that is where the tensile stress from the rarefaction wave is at its maximum, ${ }^{13}$ similar to cavitation.

This model also works well for semiconductors such as $\mathrm{Si}$, $\mathrm{Ge}$, or GaAs, where the absorption depth for $800 \mathrm{~nm}$ laser light is several microns at low intensities. This is because the first optical cycle of a high-intensity pulse can promote a sufficient number of electrons to the conduction band to convert the material to a state more like a metal. This is the physical basis for the phenomena called bandgap closure. ${ }^{14}$ Hence the interaction volume for absorption in semiconductors will quickly collapse to the skin depth of the newly formed "metal."

This process of liquid spallation is a dominant mechanism of material removal for metals and semiconductors at fluences close to the material removal threshold. Other mechanisms include phase explosion, ${ }^{15}$ Coulomb explosion in dielectrics, ${ }^{16,17}$ and even mechanical exfoliation of 2D materials (see the Yoo et al. article in this issue). In materials such as polymers, photochemistry can induce phase changes, as can irradiation of transparent oxides such as glass, where the focused intensity can induce a structural phase change or densification. ${ }^{18-20}$ This densified region has a different refractive index and can be used for guiding light. ${ }^{21}$ It can also be used to create regions where chemical attack/etching is greatly enhanced for producing microfluidic and microelectromechanical system (MEMS) devices $^{22}$ (see the Jiang et al. article in this issue).

Each of these fundamental mechanisms for material removal or transformation has allowed or enhanced a large number of applications envisaged by creative researchers around the world. Every one of these mechanisms has reached the commercial market after only 20 years of study. Yet, much work remains to be done to develop a far more detailed understanding of many of the atomistic or electronic processes involved. There are likely still many more mechanisms that have not even been discovered yet.

Much of the potential of ultrafast laser-solid interactions exists in understanding the material response mechanisms that occur when materials are pushed into extreme conditions. This largely unexplored area of materials processing is the focus of many of the articles in this theme issue. In their article, Shugaev et al. include advances in simulation and modeling of materials with atomistic and continuum methods. These are already providing insights into the role that early time scale interactions play in eventually driving a cascading set of material responses. This is also illustrated in the Abere et al. article on surface morphological modification in metals, polymers, 
and semiconductors where incubation effects alter absorption, and an accumulation of defects leads to massive rearrangement of near-surface atoms, creating morphological changes without ablation or melting. Continued research into how materials absorb energy on these short time scales and how these materials respond to the extreme conditions represent a significant opportunity to synthesize and process materials. Not always appreciated is what "extreme" means in the context of materials science.

\section{Extreme shock, extreme heating rates, extreme quenching}

Ultrafast laser-induced shock processing started more than 15 years ago with seminal work in chemistry that used shock waves to synthesize organic compounds. ${ }^{23,24}$ While this excellent work is not covered in this issue, it should be clear from the articles that we have greatly enhanced our fundamental understanding of energy transfer to materials. We now have more advanced computational capabilities as well as emerging time-resolved experimental techniques available that work well on the ps time scale of interest for pressure-induced phenomena.

Ultrafast laser interactions with materials can drive shock waves that have unprecedented peak pressures in a material, using tabletop experiments. Blast waves with $800 \mathrm{GPa}$ can be generated with a $1 \mathrm{~W}$ average power laser. While these are not useful for certain types of research (e.g., gas gun and projectile impact studies), because the shock waves are only a few ps wide, they can be applied to understand the collateral damage that occurs during ultrafast machining. Not only does the peak pressure exceed the Young's modulus in nearly every solid, the strain rates approach the highest that can be produced. This alone offers an exciting opportunity to modify materials using these extreme conditions.

Ultrafast laser-induced shock has recently been shown to represent an alternative approach to swift heavy ion (SHI) accelerators for studying radiation damage in materials from fissionable particles. ${ }^{25}$ This represents a new direction for ultrafast laser material research with the ability to use pumpprobe methods to study, for instance, the dynamics of how complex oxide materials, of importance to nuclear fuel cladding applications, undergo phase transformations resulting from defect accumulation, dislocation-driven transformation, and pressure-induced phase transformation. ${ }^{25}$ Ultrafast laser interactions with materials are an excellent match for the energy deposition per nanometer and the interaction time scale of swift heavy ions. While it will only be a useful surrogate technique and require careful SHI studies, it offers an opportunity to explore a much wider range of materials at lower cost.

In the context of "extreme heating and quenching," many in the materials community consider $10^{4}$ or $10^{5}$ degrees per second heating/quenching rates to be extreme. Several of the articles in this issue demonstrate that ultrafast melting can heat materials as much as $10^{12}$ degrees per second. This also leads to extreme strain rates as mentioned earlier. The other opportunity that ultrafast laser processing of materials offers is that the excitation depth is shallow compared to the thermal diffusion depth driven by longer pulses. When a thin layer of material that sits on a high thermal conductivity substrate is heated well above the melting point, rapid quench rates can be achieved. Shugaev et al. discuss achieving quench rates on the order of $10^{12}$ degrees per second.

In fact, recent work ${ }^{26}$ has shown that these high heating and quench rates may push materials into the vapor dome, a region in the density versus temperature phase diagram where liquid and vapor coexist. The top of the vapor dome, sometimes called the critical phase-separation region, is an interesting part of this phase field, because that is where one needs to drive a material to access phase explosion. In the vapor dome near the critical point, the liquid phase is thermodynamically unstable and will homogeneously nucleate vapor in ps. ${ }^{15}$

This work ${ }^{26}$ suggests that if one can drive a material into the vapor dome via extreme heating rates and then drive it out again via extreme quenching rates, nucleation of gas bubbles at a liquid-solid interface is induced, resulting in novel material removal mechanisms. This is illustrated in Figure 1. Here, postmortem characterization, time-resolved microscopy, and hydrodynamic simulation indicate that removal from the metal-substrate interface occurs by rapid heterogeneous nucleation and growth of vapor near the time the irradiated metal enters the vapor dome. It was speculated that vapor bubbles form without kinetically limited nucleation, similar to spinodal decomposition. Forcing materials into these exotic phase fields offers novel processing opportunities.

\section{Opportunities for transparent materials and organic materials}

Nonlinear interactions of ultrafast lasers with transparent materials, especially glasses, due to the extremely high peak intensity, have seen a significant level of interest and commercialization. This distinct feature offers three different schemes in 3D micro- and nanoprocessing, subtractive, undeformative, and additive processing. ${ }^{27}$ As described in the article by Jiang et al. in this issue, several products are available that take advantage of ultrafast laser 3D densification that occurs in glasses that allow these regions to etch far more quickly than others. This has spawned the field of glass MEMS devices. Each year, we see new and interesting results in this area, from $3 \mathrm{D}$ generation of layered structures via a Fabry-Pérot mechanism, ${ }^{28}$ to the kinds of applications discussed in the article by Jiang et al.

Much work has also taken advantage of fluence-controlled multiphoton photochemistry to produce 3D structures using a scanning focused ultrafast $\operatorname{laser}^{29}$ (see the Jiang et al. and Stratakis et al. articles in this issue). The ability to work with biological materials using these mechanisms has led to the use of ultrafast lasers in tissue engineering ${ }^{30}$ and other emerging fields. Using ultrafast lasers to process carbon-based materials such as graphene is also well reviewed (see Yoo et al. in this issue) and is an important current area of research. 


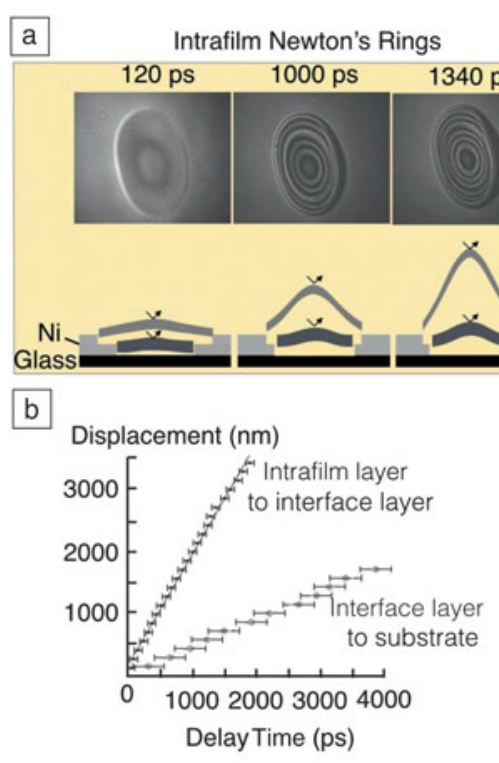

Figure 1. Very high heating and quench rates may push materials into the vapor dome, a region in the density versus temperature phase diagram where liquid and vapor coexist. (a) Two Newton's ring patterns after laser irradiation indicate removal in two distinct layers. Schematics of the moving layers are shown at each delay (not to scale). The reflections forming Newton's rings are indicated by angled arrows. (b) Displacement versus time is shown for both intrafilm and interface removal for an absorbed fluence of $0.25 \pm 0.02 \mathrm{~J} / \mathrm{cm}^{2}$. The top, faster-moving layer is removed through liquid spallation. Postmortem characterization and the velocity of the bottom layer are consistent with removal by heterogeneous nucleation of vapor However, both films are removed and reach constant velocity within 50 ps. (c) A schematic of the different paths that an ultrathin $\mathrm{Ni}$ film may take after irradiation suggests that molten $\mathrm{Ni}$ may briefly enter the vapor dome in the Ni temperature-density phase diagram. The bottom path shows intrafilm removal where the liquid layer enters and exits the vapor dome; the left branch of this path indicates vapor formed by cavitation separating the top layer. The top path is representative of liquid in the conditions for interface removal, the metal enters the vapor dome below, but near, the critical phase-separation region, and is briefly in the spinodal-like region. Note: ps, picoseconds.

\section{The challenges}

Ultrafast laser synthesis and processing of materials is an emerging field. Fundamental details of the dynamics of interaction are still elusive at many time and length scales. Yet, new experimental, theoretical, and computational methods promise new ways to address this challenge. The future of ultrafast lasersolid interactions may well depend on how the community of researchers is able to combine lasers with some of these new tools. This, of course, is challenging.

New quantum molecular dynamics (MD) approaches ${ }^{31}$ to study the early time scales are now possible with ever faster processing. So too is our ability to use MD to extend our study to larger time scales (see Shugaev et al. in this issue). Coupling the laser field and accounting for the myriad of challenging physics that results remains a challenge — but one that is being addressed every day. Perhaps some of the most exciting opportunities to address the challenge will come from the highfield ultrafast laser community. High-intensity relativistic laser research has made it possible to create ultrashort X-ray pulses via wakefield electron acceleration that are brighter than fourthgeneration synchrotrons. ${ }^{32,33}$ The entire field of attosecond science may revolutionize our ability to study materials on sub-fs time scales, subnanometer length scales, and even study the dynamics of electronic structure on fs time scales. ${ }^{34,35}$

As we discover new phenomena experimentally and theoretically that may have an impact on real-world applications, we have additional challenges. Ultrafast lasers are currently expensive and do not have the high average power that is needed for commercial manufacturing. There are two significant aspects of this problem that the articles in this issue address. The first is that without high payoff applications, there will not be the will by companies or funding agencies to invest in developing the kind of lasers that are needed. This research community must continue to be creative and innovative with our exploitation of the new physics emerging in our laboratories. The second is that to develop high average power lasers requires new technology. The latter challenge is addressed in the article by Mottay et al. in this issue, including the leading edge of fiber and disc laser approaches to building high average power fs lasers. There is a clear roadmap to building kilowatt average power lasers for manufacturing. As long as the community can continue to discover and invent high payoff applications, these lasers will become a reality.

\section{The vision}

Gérard Mourou, one of the inventors of the chirped pulse amplifier that enabled much of what is described in the articles in this issue has often said that "research is the locomotive that drives technology." Ultrafast laser material synthesis and processing of materials is a perfect illustration of that maxim. Perhaps the most exciting thing about the field is that there continues to be a wealth of new fundamental research in ultrafast fiber and disc lasers, attosecond science, extreme high field science with the emergence of the Extreme Light Infrastructure Project in Europe that is building an exawatt laser, ${ }^{36,37}$ and even the early zeptosecond-zetawatt efforts. ${ }^{38}$ The future of this field is very bright indeed.

\section{Acknowledgments}

S.M.Y. acknowledges contributions from B. Torralva and K. Schrider. S.M.Y. also acknowledges support from the Air Force Office of Scientific Research under Award Number FA9550-12-1-0465. C.P.G. is supported by the National Science Foundation under the Scalable Nanomanufacturing Grant EEC-1449305.

\section{References}

1. D.G. Cahill, S.M. Yalisove, MRS Bull. 31, 594 (2006).

2. T. Juhasz, F.H. Loesel, R.M. Kurtz, C. Horvath, J.F. Bille, G. Mourou, IEEE J. Sel. Top. Quantum Electron. 5, 902 (1999). 
3. C.P. Grigoropoulos, D.J. Hwang, A. Chimmalgi, MRS Bull. 32, 16 (2007).

4. M.J. Abere, B. Torralva, S.M. Yalisove, Appl. Phys. Lett. 108, 153110 (2016). 5. H. Rabitz, R.D. Vivie-Riedle, M. Motzkus, K. Kompa, Science 288, 824 (2000).

6. E. Gamaly, A.V. Rode, B. Luther-Davies, V.T. Tikhonchuk, Phys. Plasmas 9, 949 (2002).

7. E. Gamaly, A. Rode, Prog. Quantum Electron. 37, 215 (2013).

8. S.I. Anisimov, B.L. Kapeliovich, T.L. Perelman, Sov. Phys. JETP 39, 375 (1974).

9. B.J. Siwick, J.R. Dwyer, R.E. Jordan, R.J.D. Miller, Science 302, 1382 (2003).

10. A.M. Lindenberg, J. Larsson, K. Sokolowski-Tinten, K.J. Gaffney, C. Blome, 0. Synnergren, J. Sheppard, C. Caleman, A.G. MacPhee, D. Weinstein, D.P. Lowney,

T.K. Allison, T. Matthews, R.W. Falcone, A.L. Cavalieri, D.M. Fritz, S.H. Lee, P.H. Bucksbaum, D.A. Reis, J. Rudati, P.H. Fuoss, C.C. Kao, D.P. Siddons, R. Pahl, J. Als-Nielsen, S. Duesterer, R. Ischebeck, H. Schlarb, H. Schulte-Schrepping, T.H. Tschentscher, J. Schneider, D. von der Linde, 0. Hignette, F. Sette, H.N. Chapman, R.W. Lee, T.N. Hansen, S. Techert, J.S. Wark, M. Bergh, G. Huldt, D. van der Spoel, N. Timneanu, J. Hajdu, R.A. Akre, E. Bong, P. Krejcik, J. Arthur, S. Brennan, K. Luening, J.B. Hastings, Science 308, 5720 (2005).

11. C.W. Siders, A. Cavalleri, K. Sokolowski-Tinten, C.S. Tóth, T. Guo, M. Kammler, M. Horn von Hoegen, K.R. Wilson, D. von der Linde, C.P.J. Barty, Science 286, 1340 (1999).

12. D. von der Linde, K. Sokolowski-Tinten, Appl. Surf. Sci. 154, 1 (2000)

13. D.A. Reis, K.J. Gaffney, G.H. Gilmer, B. Torralva, MRS Bull. 31, 601 (2006).

14. E.N. Glezer, Y. Siegal, L. Huang, E. Mazur, Phys. Rev. B Condens. Matter 51, 6959 (1995).

15. M.E. Povarnitsyn, T.E. Itina, M. Sentis, K.V. Khishchenko, P.R. Levashov, Phys. Rev. B Condens. Matter 75, 235414 (2007)

16. C. Cornaggia, M. Schmidt, D. Normand, J. Phys. B At. Mol. Opt. Phys. 27. L123 (1994).

17. R. Stoian, D. Ashkenasi, A. Rosenfeld, E.E.B. Campbell, Phys. Rev. B Condens. Matter 62, 13167 (2000)

18. R.R. Gattass, E. Mazur, Nat. Photonics 2, 219 (2008).

19. L. Shah, F. Yoshino, A. Arai, S. Eaton, H. Zhang, S. Ho, P.R. Herman, Proc. SPIE 5714, 253 (2005).

20. K. Itoh, W. Watanabe, S. Nolte, C.B. Schaffer, MRS Bull. 31, 620 (2006).

21. K.M. Davis, K. Miura, N. Sugimoto, K. Hirao, Opt. Lett. 21, 1729 (1996).

22. A. Marcinkeviius, S. Juodkazis, M. Watanabe, M. Miwa, S. Matsuo, H. Misawa,

J. Nishii, Opt. Lett. 26, 277 (2001)

23. D. Dlott, M. Fayer, J. Chem. Phys. 92, 3798 (1990).

24. A. Tokmakoff, M. Fayer, D. Dlott, J. Phys. Chem. 97, 1901 (1993).

25. D.R. Rittman, C.L. Tracy, A.B. Cusick, M.J. Abere, B. Torralva, R.C. Ewing,

S.M. Yalisove, Appl. Phys. Lett. 106, 171914 (2015).

26. K.J. Schrider, B. Torralva, S.M. Yalisove, Appl. Phys. Lett. 107, 124101 (2015).

27. K. Sugioka, Nanophotonics 5, 17 (2016).

28. K. Kumar, K.K. Lee, J. Li, J. Nogami, N.P. Kherani, P.R. Herman, Light Sci. Appl. 3, e157 (2014).

29. S. Maruo, S. Kawata, "Micro Electro Mechanical Systems," Proc. IEEE 10th Annu. Int. Workshop (Nagoya, Japan, 1997), pp. 169-174.

30. M.B. Applegate, J. Coburn, B.P. Partlow, J.E. Moreau, J.P. Mondia, B. Marelli, D.L. Kaplan, F.G. Omenetto, Proc. Natl. Acad. Sci. U.S.A. 112, 12052 (2015)

31. R.E. Allen, T. Dumitrică, B. Torralva, in Ultrafast Physical Processes in Semiconductors, K.T. Tsen, Ed. (Academic Press, New York, 2001).

32. A.G.R. Thomas, K. Krushelnick, Phys. Plasmas 16, 103103 (2009).

33. A.G.R. Thomas, Phys. Plasmas 17, 056708 (2010).

34. J. Itatani, J. Levesque, D. Zeidler, H. Niikura, H. Pépin, J.C. Kieffer, P.B. Corkum, D.M. Villeneuve, Nature 432, 867 (2004).
35. Z. Chang, P.B. Corkum, S.R. Leone, J. Opt. Soc. Am. B 33, 1081 (2016)

36. T. Tajima, B.C. Barish, C.P. Barty, S. Bulanov, P. Chen, J. Feldhaus, J. Hajdu, C.H. Keitel, J.-C. Kieffer, D.-K. Ko, W. Leemans, D. Normand, L. Palumbo, K. Rzazewski, A. Sergeev, Z.-M. Sheng, F. Takasaki, M. Teshima, AIP Conf. Proc. 1228, 11 (2010).

37. G.V. Dunne, Eur. Phys. J. D 55, 327 (2009)

38. G. Mourou, T. Tajima, Eur. Phys. J. Spec. Top. 223 (6), 979 (2014).

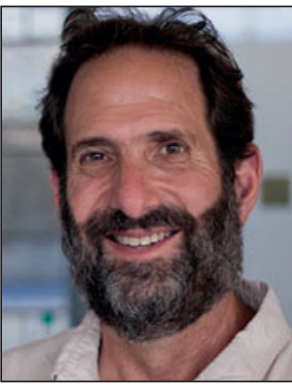

Steven M. Yalisove is a professor of materials science and engineering at the University of Michigan. He obtained his PhD degree in materials science and engineering at the University of Pennsylvania in 1986. He joined the Michigan faculty in 1989 after a postdoctoral appointment at Bell Laboratories. He has made important contributions to the fields of surface science, thin-film growth, evolution of thin-film morphology, and the interaction of high-intensity femtosecond laser pulses with materials. Yalisove can be reached by phone at 734-764-4346 or by email at smy@umich.edu.

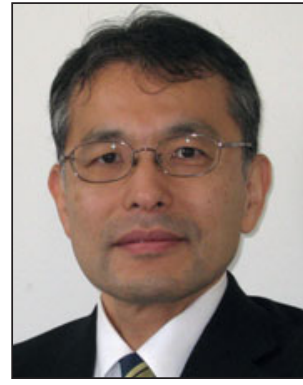

Koji Sugioka is a unit leader of the RIKENSIOM Joint Research Unit at the RIKEN Center for Advanced Photonics, Japan. He is also a guest professor at Kyoto University and Tokyo Denki University. He received his BS degree in 1984, Ms. Eng. degree in 1986, and Dr. Eng. degree in 1993, all from Waseda University, Japan. His research interests include ultrafast laser processing for microfluidic, optofluidic, microelectronic, and optoelectronic applications. $\mathrm{He}$ is a board member of the Laser Institute of America and the Japanese Laser Processing Society, and a Fellow of SPIE and OSA. Sugioka can be reached by email at ksugioka@riken.jp.

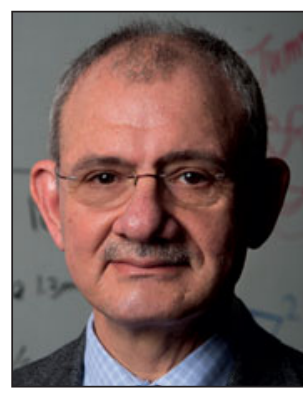

Costas P. Grigoropoulos is a professor in the Department of Mechanical Engineering at the University of California, Berkeley. He received his diploma degrees in naval architecture and marine engineering (1978), and in mechanical engineering (1980) from the National Technical University of Athens, Greece. He holds a MSc degree (1983), and a PhD degree (1986), both in mechanical engineering from Columbia University. His current research interests include laser materials processing, micro-nanomanufacturing, and nanoengineering. He is a Fellow of ASME and SPIE and editor of the International Journal of Heat and Mass Transfer. Grigoropoulos can be reached by phone at 510-642-2525 or by email at cgrigoro@berkeley.edu.

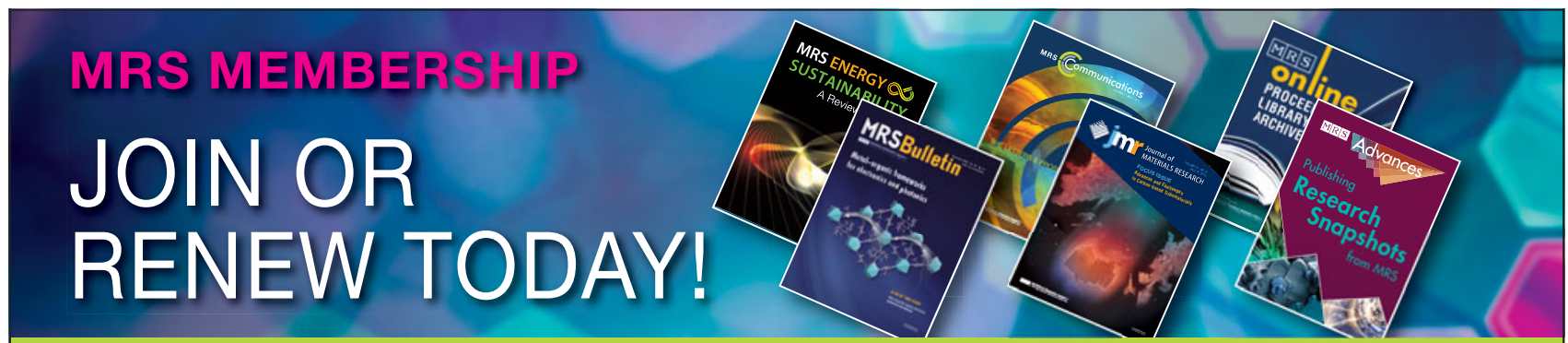

Your MRS Membership now includes online access to ALL MRS journals. www.mrs.org/membership 